\title{
Movilización de recursos del Empresariado Étnico en Almería: la importancia de las redes sociales
}

\author{
Ángeles Arjona Garrido y Juan Carlos Checa Olmos, Universidad de Almería ${ }^{1}$
}

\begin{abstract}
In the county of Almería (Spain) the labor incorporation of the non-Communitarian foreigners is a reality since the expansion of the agricultural production system based in hothouses, by the middle of $80 \mathrm{~s}$. This labor incorporation in the agriculture is based on precariousness and uncertainty. Some non-Communitarian foreigners have opted, after years of stay and drawing an enormous quantity of obstacles, to start up a self-employed business. In their project underlies the desire of getting a bigger labor and personal stability, as well as a future economic improvement. Now then, to be able to install a business, the immigrant must mobilize all the resources available. In this work we make a review for those resources moblized by the immigrant, where personal networks plays a main role: family, friends and ethnic solidarity.
\end{abstract}

Key words: Immigration, work market, class resources, ethnic resources, personal networks, ethnic solidarity.

\section{Resumen}

En la provincia de Almería (España) la incorporación laboral de los inmigrados extracomunitarios es una realidad desde que se extendió el sistema de producción agrícola en invernadero, a mediados de los 80. Esta incorporación laboral en la agricultura se ha producido en un segmento del mercado sometido a precariedad e inestabilidad. Algunos extranjeros no comunitarios han optado, después de años de estancia y sorteando una enorme cantidad de obstáculos, por instalar un negocio por cuenta propia. En su proyecto subyace el deseo de conseguir una mayor estabilidad laboral y personal, así como una mejora económica de futuro. Ahora bien, para llegar a instalar un negocio, el inmigrado ha de movilizar todos los recursos a su alcance. En este trabajo hacemos un repaso por aquellos recursos que el inmigrado dispone entre los que juega un papel principal la red personal: la familia y amigos en la emigración y la solidaridad étnica.

Palabras clave: Inmigración, mercado de trabajo, recursos de clase, recursos étnicos, red personal, solidaridad étnica.

\section{I ntroducción}

En las sociedades económicamente avanzadas la incorporación de mano de obra extranjera a sus mercados de trabajo es ya una realidad generalizada, “la demanda de inmigrantes está incrustada de manera estructural en muchas sociedades. La globalización parece aumentar el número de trabajos indeseables y precarios" (Stalker, 2000: 133). España, país de

\footnotetext{
${ }^{1}$ Enviar correspondencia a: arjona@ual.es
} 
reciente adhesión a la entrada de flujos migratorios y enmarcado dentro del campo migratorio europeo, se caracteriza por la concentración de los inmigrados en determinadas áreas geográficas, bajo la lógica de la estructuración de unos mercados de trabajo muy especializados agricultura, construcción, servicios. La provincia de Almería destaca, en este sentido, por una alta presencia de nuevos inmigrantes ${ }^{2}$ que se emplean, mayoritariamente, en la agricultura intensiva, aunque también podemos encontrar a inmigrados ocupados en la industria auxiliar agrícola, construcción, venta ambulante o servicio doméstico. En cualquier caso, todos estos mercados presentan las características comunes de etnificación laboral, eventualidad, flexibilidad, precariedad, trabajadores irregulares ${ }^{3} \ldots$, en definitiva, elementos propios del mercado secundario (véase Piore, 1974).

Algunos extranjeros no comunitarios han optado, después de años de estancia y sorteando gran cantidad de obstáculos ${ }^{4}$, por instalar un negocio por cuenta propia 5 . En su proyecto subyace el deseo de conseguir una mayor estabilidad laboral, así como una mejora económica que los mercados de trabajo de la provincia no le ofrecen. Ahora bien, para conseguir instalar un negocio el inmigrado tiene que movilizar y activar todos los recursos de los que dispone. En este trabajo, por tanto, vamos a tratar de analizar los elementos y factores que los inmigrados tienen que utilizar para convertirse en emprendedores étnicos. Para ello, seguimos, de un lado, las directrices de las investigaciones llevadas a cabo en Estados

\footnotetext{
2 Utilizamos el concepto nueva inmigración para describir el fenómeno de la migración extracomunitaria que se emplea en las agriculturas mediterráneas (véanse Hoggart y Mendoza, 1999; Mottura, 1992; Pugliese, 1992). La nueva agricultura se ha convertido en uno de los sectores que actúan como polo de atracción de migrantes, hecho que lo diferencia de las migraciones anteriores fordistas. La nueva migración se dirige a países, provincias o zonas que anteriormente habían sido expulsoras de mano de obra, caso de Almería. También, la incorporación de estos inmigrantes invierte la dirección habitual de los flujos desde el campo a la ciudad.

3 Diversas investigaciones, para éste y otros contextos españoles, ponen de relieve la ocupación de nichos laborales por parte de los inmigrados, que la mayoría de los nacionales no ocuparían (véanse, entre otros, Martínez, García y Maya, 1996; Colectivo Ioé, 1995a; 1995b; 1998; Avellá, 1995; Checa, 1995; Cachón, 1997). Son trabajos que se caracterizan por alta peligrosidad, con jornadas laborales por encima de las ocho horas, cobrando sueldos inferiores al salario mínimo interprofesional, etc.

${ }^{4}$ Se trata de dificultades desde un punto de vista jurídico, social y económico (véanse Trinidad, 2003; Arjona, 2004a).

${ }^{5}$ Para profundizar sobre la instalación de negocios étnicos en la provincia de Almería véase Arjona (2004b).
} 
Unidos y Europa ${ }^{6}$, distinguiendo entre recursos de clase - capital financiero, capital humano, capital cultural y capital social- y recursos étnicos -créditos étnicos, sociedades de préstamo y fuentes de capital de tamaño más pequeño-. Y, de otro, partimos de la idea que las redes proporcionan soluciones laborales cuando los inmigrados están en situación de desventaja, por ello, son los intermediarios de la misma nacionalidad los que llegan a dominar la incorporación de sus co-nacionales en un determinado sector laboral ${ }^{7}$ (Macdonald y Macdonald, 1964), a la vez que pueden actuar de estímulo para la creación de micro empresas.

De lo que se trata, en definitiva, es mostrar por un lado, si el apoyo proporcionado por las redes en destino es un elemento clave a la hora de explicar la creación de empresas regentadas por inmigrados, que, a la postre, tampoco tendrán éxito sin la presencia anterior de una comunidad inmigrada. Y, por otro, si estamos ante un proceso de proliferación de economías étnicas.

\section{Marco conceptual}

La teoría de las redes sociales mantiene que el inmigrado, en una situación de mercado de trabajo segmentado, recurre a las redes sociales, incluso desde el país de origen, para, en la medida de lo posible, subsanar la situación de falta de información, discriminación o abandono administrativo. Por ello, el análisis de las redes nos puede explicar algunas particularidades de la comunidad inmigrante.

Las investigaciones en este campo se empezaron a abordar en los años setenta del siglo pasado, aunque ha sido en los últimos años cuando esta perspectiva ha cobrado mayor relevancia, poniendo énfasis en el análisis de las relaciones que se establecen entre los actores y el sistema social en el que participan ${ }^{8}$. Con el concepto de red aparece una nueva forma de

\footnotetext{
${ }^{6}$ Son múltiples las investigaciones y el corpus teórico desarrollado en esta temática, véanse, entre otros, los trabajos de Bonacich (1979, 1993); Light (1971, 1972); Ma Mung (1994), Light y Gold (2000); Kloosterman (2000); Kloosterman y Rath (2001).

7 En España se produce, fundamentalmente, con determinados negocios de las comunidades chinas, situados en diferentes zonas de la geografía: Lavapiés en Madrid, Ciudad Vella en Barcelona, Delicias en Zaragoza, Elche en Alicante. Véanse, entre otros, los trabajos de Beltrán (2000) Cebrián y Bodega (2004).

${ }^{8}$ En España destacan los trabajos de Maya (1999; 2002) y Molina (1995; 2001), entre otros.
} 
describir la estructura de los fenómenos sociales, en cuanto representa un nexo de unión entre la acción individual y la acción colectiva, entre la personalidad y la sociedad, entre el análisis macro y micro. Lo que se traduce en que "a diferencia de los análisis tradicionales que explican, por ejemplo, la conducta en función de la clase social y la profesión, el análisis de redes se centra en las relaciones y no en los atributos o los elementos" (Molina, 2001: 13). Los últimos estudios a partir del trabajo de Ronald Burt, además de analizar la inserción laboral desde una perspectiva del capital humano, introducen el capital social, que viene dado por la aplicación del análisis de redes sociales al networking. Para Molina (2001:54) “Burt abunda en la idea del capital social como complementario del capital humano. Si el capital humano está constituido por el conjunto de aptitudes y saberes adquiridos por la educación, la experiencia y la formación continua, el capital social está constituido por las personas que nos conocen, que saben cómo somos y de lo que somos capaces (...). La contribución específica de Burt es la idea de que las redes personales se pueden gestionar, manipular, de forma que nuestra posición conecta grupos desconectados, lo cual nos confiere más poder e influencia, es decir, maximizar nuestro grado de intermediación, nuestra capacidad de ser puentes en la red, de conectar grupos solamente a través de nosotros".

La red social se define, de este modo, como una estructura que puede no ser perfectamente visible, pero que es real. En la red está inserto un individuo o un grupo de personas, que se construye por las relaciones que éstas mantienen entre sí y con otras que están fuera del grupo (Speck y Attneave, 1974). Y, como sostiene Requena (1991), la red social está compuesta por un actor -individual o colectivo- y por todos sus familiares, amigos, parientes, compañeros, conocidos y otros que pueden ofrecer ayuda significativa, tanto en intercambio de información como de bienes y servicios.

Alejandro Portes (1998) considera la migración como un fenómeno colectivo más que individual, para ello introduce el concepto de red social migratoria. Las personas que emigran a un determinado destino lo hacen porque tienen a otras personas como punto de referencia-cabezas de puente-, con quienes intercambian información y ayuda, la migración se conforma así 
como un fenómeno en el que los individuos actúan de forma interrelacionada. Así pues, se puede definir la red social migratoria como el conjunto de relaciones sociales informales, entre los migrados y los potenciales migrantes, que unen las dos sociedades, la emisora y la receptora. A su vez, estas relaciones se insertan en un sistema migratorio "dotado de cierta vocación de permanencia y acompañado de un denso tejido de interrelaciones de diversos órdenes, que se establece entre una región receptora de inmigración y un conjunto de países emisores de emigración" (Arango, 1993:26). La mano de obra migrante circula por un mercado laboral mundial, pero éste es imperfecto, cobrando entonces las redes sociales gran efectividad como sistema de asignación ocupacional. Como apunta Herranz (1996:58) “las redes de relaciones entre los inmigrantes no sólo intervienen en la decisión de emigrar a un lugar determinado, sino en la conexión de la oferta en el país emisor con la demanda en el país receptor".

Siguiendo esta línea, y puesto que los mercados de trabajo se encuentran fragmentados -limitados por barreras institucionales, geográficas, jurídicas y sociales-, la relación entre oferta y demanda está sometida a muchos condicionantes, ya que no se produce una competencia perfecta, ni libre. Con arreglo a estas limitaciones la movilidad laboral es muy costosa, de esta forma "la respuesta social al coste de movilidad es la utilización de relaciones sociales (amistad, parentesco, conocidos, etc.) como cauces de búsqueda y acceso al empleo que reducen los costes de la misma" (Requena, 1991: 100).

En consecuencia, las redes sociales no sólo abaratan los costes de la incorporación al mercado laboral, también adjudican posiciones en la estructura ocupacional que, de alguna forma, no se satisface con los canales oficiales. De hecho, es muy común que el acceso a la primera oferta de empleo se lleve a cabo a partir de la red. Al inmigrado le es más útil la red para encontrar empleo que mediante los cauces normalizados ${ }^{9}$.

\footnotetext{
${ }^{9}$ Los anuncios en prensa, la presentación de currículos, las empresas de trabajo temporal o las agencias de colocación no son cauces efectivos de incorporación laboral para los inmigrantes, entre otras razones, porque los desconocen, bien por las barreras idiomáticas, bien porque pertenecen a redes sociales cerradas, e incluso, por discriminación.
} 
Por otro lado, la red de relaciones se hace más fuerte conforme se estrechan los lazos entre actores, está en función del tiempo que haya transcurrido en su relación, de la intimidad, de la intensidad emocional, así como de los servicios recíprocos que caracterizan la relación. Cuando se trata de buscar empleo se establecen largas cadenas de lazos no muy fuertes, pero que resultan de gran utilidad. En este sentido, Granovetter (1973) anuncia que antes de la migración pueden existir lazos débiles y una vez en destino estos lazos se hacen más fuertes.

Otro elemento importante que determina la utilización de la red es la actitud y comportamiento hostil que tiene el contexto de recepción ${ }^{10}$ (véase Portes y Böröcz, 1992) frente a los migrantes. La interacción intensa dentro de un medio extraño común y la formación de organizaciones e instituciones de inmigrantes refuerzan la identidad étnica e intensifican la sensación de comunidad y de pertenencia al grupo. Surge así la conciencia de grupo étnico o la etnicidad y se intensifican las conexiones sociales entre los miembros (Waldinger, Aldrich y Ward, 1990). El contexto de recepción produce, en muchas ocasiones, una concentración espacial que desemboca en la formación de enclaves. Lugares donde el colectivo de inmigrados reproduce el sentido de comunidad y promueve la autoidentificación con el grupo, así como la solidaridad étnica (Waldinger, Aldrich y Ward, 1990).

La conjunción de factores: transnacionalismo, concentración espacial, utilización de redes y solidaridad étnica va a favorecer, frente a la hostilidad del contexto, la aparición de economías étnicas. En este sentido, cuando Bonacich y Modell (1980) desarrollaron el concepto de economía étnica, intentaron diferenciarlo del empleo generado dentro de la economía general y global, considerado como el empleo que la minoría crea para sí misma ${ }^{11}$. La economía étnica, por tanto, incluye cualquier persona inmigrante que sea empleador, autoempleador o que esté empleado en empresas co-étnicas. “El contorno de una economía étnica está definido por raza, etnicidad u

\footnotetext{
${ }^{10}$ La hostilidad de los autóctonos con los inmigrados explica, en parte, la solidaridad étnica, que a la postre tendrá influencia cara al éxito o fracaso de las economías étnicas. Las concentraciones de inmigrados en el ámbito residencial, ocupacional, esparcimiento o reunión, permiten la creación e intensificación de lazos y, por tanto, la eficacia de la red para acceder al empleo étnico o hacer uso de los servicios como cliente.
} 
origen nacional, caracterizándose por alcanzar ventajas en las relaciones entre propietarios de negocios y entre propietarios y trabajadores del mismo origen nacional" (Logan, Alba y McNulty, 1994: 698).

Determinados autores han sostenido que las economías étnicas crean oportunidades para los trabajadores inmigrados, debido a la movilización de lazos étnicos que se emplean en la búsqueda de oportunidades en el mercado, sobre todo, si las redes están concentradas en enclaves étnicos o eventualmente distribuidos a través del espacio (Light y Bonacich, 1988; Waldinger, 1993; Logan, Alba y McNulty, 1994) $)^{12}$.

Además, la economía étnica funciona, en ocasiones, como mercados de trabajo internos, a fin de proteger a los inmigrados de la competencia del mercado de trabajo general, mientras adquieren las habilidades para montar su propio negocio (Bailey y Waldinger, 1991; Waldinger, 1994, Jiobu 1998), hasta tal punto que las economías étnicas actúan como una "escuela de emprendedores" (Light y otros, 1994). Presumiblemente el empleo entre empresas de coétnicos promociona el autoempleo, ya que dotan de habilidades para crear un negocio propio y enseñan qué hay que hacer para que éste funcione (Waldinger, 1985; Portes y Bach, 1985; Portes y Manning, 1986).

En la economía étnica el empresario tiene una posición intermedia, mezcla de éxito económico y escaso poder político, por eso se necesita utilizar la red social. Con la utilización de la red social se generan una serie de ventajas para el grupo, gracias a su capital social, (Coleman, 1988; Osterman, 1976) o al capital relacional (Requena, 1991). Los bienes relacionales van a estar en función de la posición más o menos estratégica que el individuo tenga en la red social. El acceso a determinados recursos, a partir del capital social, es muy importante, por ejemplo, se pueden acceder a ayudas económicas destinadas a inmigrantes, contactar con asociaciones pro-inmigrantes y de inmigrantes a fin de obtener información, asesoramiento, ayuda legal.

\footnotetext{
11 Light y Karageorgis (1994:647) definen también economía étnica como "los autoempleados y
} empleadores, sus trabajadores familiares no asalariados y sus empleados coétnicos". 
Queda claro, por tanto, que debemos conocer el capital social del que disponen y utilizan los inmigrados para entender sus procesos de inserción laboral en la nueva sociedad. Recurso que habría que añadir a su capital humano, desarrollado en relación al contexto de recepción.

\section{Metodología y datos}

Teniendo en cuenta las variables descritas anteriormente, la hipótesis de partida de esta investigación son dos. En primer lugar, para la creación de economías étnicas el inmigrado ha de movilizar todos los recursos - de clase y étnicos- de los que dispone, especialmente, la utilización del capital social, estructurado en torno a los lazos fuertes -familia y amigos- y los lazos débiles - coétnicos. En segundo lugar, el mantenimiento y consolidación de las economías étnicas están fundamentadas en la solidaridad étnica, esto es, se abastecen de empleados y clientes coétnicos.

Las estadísticas oficiales sobre empresas con titulares extranjeros como son el Impuesto de Actividades Económicas, las Licencias de Apertura de los diferentes Ayuntamientos o el Alta en la Seguridad Social en el Régimen Especial de Trabajadores Autónomos, que ofrece el Ministerio de Trabajo, presentan graves deficiencias, por ejemplo, no registran las empresas irregulares o ilegales, tampoco muestran información detallada sobre la actividad desarrollada o la nacionalidad del titular. Para cubrir estos y otros vacíos estadísticos hemos realizado un trabajo de campo exhaustivo: rastreando calle por calle las principales ciudades de recepción de inmigrantes extracomunitarios ${ }^{13}$, para hacer un recuento de los negocios abiertos, valiéndonos de varios informantes clave para acceder a locales o negocios abiertos sin declarar.

Una vez realizado el recuento de todos y cada uno de los negocios -legales e ilegales- de empresarios extracomunitarios, con excepción de los empresarios de origen chino y los profesionales liberales ${ }^{14}$, administramos

\footnotetext{
12 Light y colaboradores (1994) también demostraron que la aparición de una economía étnica no siempre tiene porqué ir asociada a concentración residencial.

${ }^{13}$ Adra, El Ejido, Vícar, La Mojonera, Roquetas de Mar, Almería (capital) y Níjar.

${ }^{14}$ Hemos excluido de nuestra investigación a los empresarios chinos por considerar que se trata de un tipo de negocio con un servicio ofertado a una clientela abierta, no exclusiva, ni enfocada a su
} 
una ficha/cuestionario donde se recogían variables de tipo sociodemográfico, laboral, residencial y de capital social. La muestra final la conforman 182 negocios sobre 206 establecimientos contabilizados.

\section{Resultados}

En este apartado vamos a describir las variables, elementos y recursos que utilizan los inmigrados emprendedores en Almería para la apertura e instalación de sus negocios. Para ello, seguimos la clasificación de Light y Gold (2000) donde se distingue entre recursos de clase y recursos étnicos. Señalaremos, para cada caso, la cuantificación e importancia que tienen para la apertura o consolidación de los negocios en Almería, para que se pueda comparar con lo sucedido en otros lugares.

\subsection{Recursos de clase}

En la creación de cualquier actividad económica y empresarial se debe disponer de un importante capital financiero, como aval de un funcionamiento inicial exitoso. Sin embargo, la propia estructura de las economías étnicas y la ocupación de un fragmento, con su propia idiosincrasia, de la economía general ha generado que los empresarios étnicos asentados en Almería hayan recurrido o solicitado muy pocas ayudas financieras - o de asesoramiento- a las entidades y organismos públicos que ofrecen dichos servicios. Así se entiende, por ejemplo, que tan sólo el 3,1\% declaran haber recibido asesoramiento, acudiendo, en este caso, a organismos como el ayuntamiento, el INEM o la Delegación de Economía de la Junta de Andalucía. De forma similar, sólo un 38,9\% de los empresarios declaran haber recibido algún tipo de ayuda económica, subvención, préstamo, bien sea de entidades financieras autóctonas, familia o compatriotas.

Estos datos señalan la importancia que ha tenido el ahorro privado en la instalación del negocio, dinero que, según declaran los emprendedores, procede, fundamentalmente, del capital obtenido a lo largo de los años en su ocupación en los mercados de trabajo de la provincia. En menor medida, 
los empresarios étnicos almerienses utilizan un capital inicial procedente de la venta de propiedades $u$ otros recursos financieros en origen.

Determinados estudios internacionales muestran, también, que la principal fuente de capital financiero es el propio, obtenido de los salarios de los empleos en otros mercados de trabajo (véanse, entre otros, Boissevain y otros, 1992 o Blashke y Ersoz, 1986). Así, por ejemplo, Light (1972) demostró para los emprendedores americanos que el $70 \%$ de los casos no necesitaron de ayudas financieras.

Ahora bien, aquellos empresarios que inicialmente han precisado de ayuda económica las fuentes donde han recurrido han sido: en primer lugar, a familiares $(24,6 \%)$; en segundo lugar, amigos, fundamentalmente $\operatorname{coétnicos}^{15}(8,5 \%)$ y, en último lugar; entidades financieras autóctonas $(5,8 \%)$. Lo que pone en evidencia la importancia de las redes más cercanas e intensas a la hora de obtener dinero por parte de los inmigrados necesitados. Como la familia y amigos se tratan de recursos étnicos, que veremos posteriormente, ahora nos centramos en el capital obtenido a través de las distintas entidades financieras.

Son una minoría los empresarios; por un lado, que han solicitado ayuda financiera a una entidad privada $y$, por otro, muy pocos a los que habiéndolo solicitado se les ha concedido. Las entidades financieras más demandadas son las sucursales de Cajamar, Caixa, Unicaja y BBVA, situadas en la provincia. Los motivos principales para negar la concesión de ayudas son la inviabilidad del negocio o la falta de aval.

Sin embargo, unido a estos condicionantes, se esconde cierta discriminación. En determinados casos los inmigrantes aun teniendo una importante garantía, bien a través de un amigo con un negocio consolidado, e incluso, algún español, estas sucursales se niegan a concederles el préstamo por el miedo a convertirse en las entidades prestatarias de los inmigrantes. Además, cuando acceden a su concesión, las condiciones son

\footnotetext{
${ }^{15}$ Algunos de ellos situados en el extranjero, con negocios establecidos.
} 
diferentes a las impuestas a otros clientes: mayor interés, más garantías ${ }^{16}$, etc. En suma, como Pierce (1974) apunta, los inmigrados procedentes de países pobres tienen grandes dificultades para acceder a préstamos financieros.

El segundo elemento de los recursos de clase es el capital humano. Por éste vamos a entender al patrimonio educativo, formativo y de experiencia que poseen los sujetos. Sin duda, éste puede ser de gran utilidad a la hora de decidir instalar un negocio. La oportunidad de ascender laboralmente también depende, en buena medida, del nivel de estudios que se posea y de la experiencia profesional alcanzada; no en vano, para el caso de los inmigrados es muy probable que la estructura del mercado de trabajo no les facilite presentar su capital humano como un aval que decida su inserción en el mismo, o le requiera mucho más tiempo poder utilizarlo (demostrarlo). Aún así, observamos que el $67,7 \%$ de los empresarios étnicos declaran tener estudios secundarios o universitarios, frente al $23,6 \%$ que sólo posen los estudios primarios y el $8,7 \%$ que no saben leer, ni escribir. Nivel educativo que, en determinados casos, es fundamental para la gestión de la empresa.

Sin embargo, dentro del capital humano el elemento fundamental para la instalación exitosa de un negocio es la experiencia profesional obtenida en el país de origen o en otros países de la Unión Europea. Para el caso almeriense el 64,2\% de los emprendedores traían una experiencia laboral previa, especialmente, se habían dedicado al comercio por cuenta propia familiar- un $32,7 \%$, el resto habían sido asalariados en otros negocios. Este hecho muestra la continuidad laboral a partir de las destrezas y habilidades adquiridas. De tal forma que todos los empresarios actuales, que también lo fueron en destino, afirmaban que su fin último era montar su propio negocio y conseguir una clientela estable. En consecuencia, los resultados del trabajo de campo muestran que los empresarios que tenían experiencia en los negocios, previa a la migración, consiguen instalar el suyo en destino en un periodo de tiempo menor.

\footnotetext{
16 Véanse, entre otros, Munnell y otros (1996) o Simon (1990) para casos parecidos ocurridos en Estados Unidos o Francia.
} 
El tercer recurso de clase, íntimamente relacionado con el anterior es el capital cultural ${ }^{17}$, entendido como los conocimientos relacionados con los idiomas, la posición social, la ética emprendedora, etc. Parece evidente, sin duda, que al capital cultural se le considere como un recurso al que, mayoritariamente, pueden acceder aquellos individuos que tienen un estatus social medio y medio-alto. A lo que también se supone que esta situación suele traducirse en esfuerzos por alcanzar puestos de trabajo deseados para mantener dicha posición. En definitiva, estos individuos están abocados a lo que se ha dado en llamar "cultura ocupacional"18.

Para aproximarnos al capital cultural que posen los inmigrados empresarios averiguamos por un lado, las profesiones de sus padres, ya que en muchos casos la cultura ocupacional se trasmite de padres a hijos. Por otro, el grado de conocimiento de idiomas de los propietarios o empleados de las empresas.

La actividad laboral más común del cabeza de familia de los emprendedores étnicos almerienses es la de comerciante $(27,2 \%)$, seguida por otros servicios $(25,9 \%)$, agricultura, pesca y ganadería $(11,2 \%)$ y, por último, administración y política $(10,5 \%)$. En cuanto al conocimiento de idiomas, resulta evidente, que para un mejor funcionamiento empresarial el dominio del idioma del país donde se reside debe ser perfecto, de esta forma le será más fácil solucionar trámites y papeleos. A su vez, el dominio de más lenguas o dialectos permite poder comunicarse con otros clientes no coétnicos: tanto españoles, como de otra nacionalidad inmigrada.

El último recurso de clase es el capital social. Los proyectos migratorios que se desarrollan en sociedades que tienen una tradición migratoria siempre

\footnotetext{
17 En este trabajo cobra sentido distinguir capital cultural y capital humano. Al primero, Bourdieu (1991:14) lo define como "una competencia en una sociedad de alto estatus cultural". Mientras que el capital humano nos ayuda incrementando nuestra propia productividad y compitiendo con otros empleados o empleadores, sin embargo, el capital cultural nos ofrece reconocimiento y prestigio social, que posteriormente se traduce en facilidades para conseguir trabajos deseables, matrimonios ventajosos y contactos de negocios.

${ }^{18}$ Berger (1991) se refiere a la cultura del emprendedor como parte del capital cultural, en este caso, una cultura ocupacional. La cultura ocupacional de los empresarios se refleja en habilidades, actitudes y valores que la burguesía empresarial necesita para introducirse en la economía de mercado. Además, esta cultura ocupacional se transmite más en la familia que en la escuela o la calle: el pariente cercano que observa esta dinámica seguro que va a obtener una socialización emprendedora, en este sentido, mejor que el resto de sus compañeros. Cuando en una familia hay tradición empresarial "se heredan" las habilidades, destrezas y conocimientos para regentar el negocio. Esto suele suponer, que cuando en origen la familia tenía tradición de comerciante, en destino van a intentar reproducirlo.
} 
son ventajosos, al menos desde el primer momento. El futuro migrante gozará, con toda probabilidad, de unas redes sociales y familiares que le abren camino: hermanos, primos, tíos, vecinos que ya se han instalado en destino. En este sentido apunta Maya (1999:71) "tener un amigo o familiar en España ayuda a aminorar los costos de oportunidad, aquellos que el individuo pierde mientras se desplaza y encuentra trabajo". Por esto, también para el empresariado étnico el capital social es sumamente importante.

Sin duda, en la creación de un negocio son fundamentales los apoyos mutuos y las ayudas familiares. Incluso una vez instalado el empresario/a intenta acelerar el proceso de reagrupación familiar, pues toda la familia en destino supone un pilar importante para la consolidación del negocio. Sobre todo, si tenemos en cuenta que el titular o encargado, en repetidas ocasiones, se ausenta para gestionar la documentación, realizar los pagos pertinentes, traer nueva mercancía, etc. Por tanto, lo ideal y deseable, para esos momentos, es dejar al cargo de la empresa una persona de total confianza.

Para el caso almeriense, los empresarios afirman en un 68,1\% que están casados, frente al $31,9 \%$ de solteros. Ahora bien, y lo más importante para los negocios, el $67,9 \%$ tiene familiares en destino -especialmente hermanos, tíos, cónyuge e hijos ${ }^{19}$-. De tal forma que en los 119 negocios en los que hay algún empleado, en 97 existe alguna relación de parentesco con el empresario: en primer lugar, hermanos, seguido de hijos y cónyuges. Ahora bien, estas relaciones no garantizan tener de un contrato laboral en regla. A veces, los empleados coétnicos - familiares o no- sólo se benefician de la rapidez para encontrar un trabajo y del aprendizaje de habilidades que le pueden servir para instalarse uno propio $^{20}$.

El número de empleados que se inserta en los negocios étnicos de Almería no es muy elevado, el $63 \%$ tiene sólo un empleado y el $31,9 \%$ a dos. Cifras

\footnotetext{
${ }^{19}$ De los ochenta y tres empresarios que declaran tener hijos, sólo catorce los tiene en origen, el resto tiene todos sus hijos en Almería.

20 Tienda y Raijman (2000:453) en un estudio con mexicanos en Norteamérica concluyen: “Ios propietarios mexicanos con experiencia previa en las empresas coétnicas consiguen adquirir más habilidades para montar sus propios negocios que aquellos coétnicos que no han trabajado en empresas de coétnicos".
} 
que nos informan sobre negocios pequeños, fácilmente atendibles con pocas personas. Además, dada la circunstancia de tener abierto todo el día, permite a los clientes acceder a sus servicios cuando le es más cómodo, de manera que no hay aglomeraciones.

En conclusión, lo realmente notorio de este caso es que sin la ayuda de los familiares y coétnicos el negocio no podría subsistir.

\subsection{Recursos étnicos}

Los recursos étnicos, como señalamos anteriormente, hacen referencia a créditos étnicos, sociedades de préstamo y fuentes de capital de tamaño más pequeño. Recursos como señalan, entre otros autores, Engstrom y McCready (1990) son fundamentales e imprescindibles para la instalación y éxito de cualquier negocio étnico, debido a su influencia en la estructura de oportunidad del inmigrado.

En Almería, el trabajo de campo nos muestra que no se utiliza ${ }^{21}$, aún, una estructura de recursos de este tipo, que tenga una importancia tan relevante como los casos norteamericanos - a partir de los gae22 o las asociaciones de crédito (ROSCAs)- y europeos -sociedades de préstamo, como la Hebrew Free Loan Association.

Por tanto, y como vimos páginas arriba, el flujo económico del que disponen los inmigrados inicialmente para la apertura del negocio son sus ahorros y

\footnotetext{
${ }^{21}$ Existen iniciativas para fomentar el autoempleo entre colectivos desfavorecidos: el Instituto de Crédito Oficial (ICO) en colaboración con el Fondo Europeo de Inversión (FEI) ha creado una línea de microcréditos dirigidos a colectivos desfavorecidos que tienen serias dificultades para acceder a la concesión de créditos bancarios por la vía ordinaria. Estos colectivos están formados por parados de larga duración, mujeres, discapacitados, inmigrantes, mayores de 45 años y hogares monoparentales. El objetivo de este tipo de microcréditos es la financiación de proyectos empresariales que fomenten el autoempleo, a través de la creación de microempresas. Definiéndolas como aquellas que cuentan con menos de diez empleados, tengan un volumen de negocio anual inferior a un millón de euros, un balance anual inferior a 1,4 millones de euros y no estén participadas en más de un $25 \%$ por una gran empresa.

En segundo lugar, y en un ámbito más local, la Consejería de Empleo y Desarrollo Tecnológico de la Junta de Andalucía ha puesto en marcha en Málaga el Programa "Emprendedores Multiculturales", iniciativa que se enmarca en los compromisos de fomento de la cultura emprendedora e integración de la población inmigrante del I Plan Integral para la Inmigración en Andalucía.

Por último, el Instituto de Fomento de Andalucía (IFA) y el Centro Andaluz de Emprendedores (CADE) pretenden apoyar la creación de empresas de emprendedores nacidos fuera de España, "mediante un programa que les ofrecerá formación específica sobre gestión empresarial, legislación española y trámites administrativos con la que mejorarán sus conocimientos y además les permitirá conseguir financiación de sus proyectos a través de microcréditos".
}

Sin embargo, y a pesar de estas iniciativas, ningún empresario étnico en Almería recurrió a ellas, bien por desconocimiento, bien por desconfianza y exceso de burocracia. 
los préstamos de familiares y amigos fundamentados en la solidaridad étnica, que tan importancia tiene para las economías étnicas (véase Bonacich y Modell, 1980; 1981).

Las condiciones del préstamo familiar son generalmente muy flexibles. En ocasiones se trata de una donación sin reposición posterior, sobre todo, entre hermanos y padres. En otras, la devolución del dinero se hace sin el cobro de un interés y en un plazo largo de tiempo, también común entre otros familiares y coétnicos.

Lo destacado del caso almeriense es la reducida frecuencia con la que se recurre a los coétnicos para recibir un préstamo económico $(8,5 \%)$, cifra similar al uso de las entidades privadas (5,8\%). En consecuencia, la escasa utilización de este recurso étnico en Almería contrasta con la utilizada por otros grupos en otros lugares. Así, por ejemplo, Bonacich (1976) para Norteamérica cifra este recurso coétnico - no familiar- tan importante como el familiar. Resultados similares muestra el caso europeo (véanse BodyGendrot y Ma Mung; 1992; Boissevain, 1992; Kloosterman, van der Leun y Rath, 1998; Light y Gold, 2000). La explicación de esta situación puede ser doble: de un lado, en Almería todavía nos encontramos ante una economía étnica escasamente desarrollada, donde los lazos empresariales son todavía escasos y el tipo de estructura que se desarrolla en torno a ellos es aún débil e individualizada; de otro, porque los inmigrados una vez que han decidido montar el negocio han hecho un cálculo de los gastos necesarios y se han provisto de ellos, mediante el ahorro personal y/o familiar.

Por tanto, y a pesar de la escasa incidencia actual de los préstamos coétnicos en Almería, no significa que éstos no tengan importancia, ni que en un futuro sea un recurso más utilizado, puesto que existen determinados indicios de que esta situación se va a producir. En primer lugar, las ayudas que están empezando a ofrecer los almacenes de servicios étnicos de venta al por mayor - caso de Agdran o Aljadida- para que se abran locales y comercialicen sus productos. En segundo lugar, las relaciones de ayuda que

\footnotetext{
22 Un gae consiste en un grupo de amigos o miembros de una organización, que contribuyen a un fondo común que después se entrega a un miembro. Lo más normal era usar este montante para abrir un nuevo negocio.
} 
se establecen entre los empresarios asentados con local y los vendedores ambulantes autónomos.

En cualquier caso, cuando se produce el préstamo de coétnicos la devolución del préstamo sí conlleva el cobro de un interés, pero el tiempo para rembolsar la cantidad es largo (más de cinco años).

En definitiva, a falta de consolidación de determinados recursos étnicos, la solidaridad étnica se convierte en el elemento clave para entender y explicar el funcionamiento actual de los negocios étnicos en Almería y de otros lugares en España. El Diccionario de la Real Academia Española define solidaridad con dos acepciones, una, como adhesión circunstancial a la causa o empresa de otros $y$, dos, como comunidad de intereses y responsabilidades. Tanto la primera, como la segunda sirven para describir la situación que se genera en torno a los negocios étnicos. Con la particularidad de darse entre personas de un mismo origen y en dos sentidos: primero, desde el empresario al empleado, donde el primero gana aceptación entre el colectivo, lo que normalmente se traduce en mayor clientela y el empleado, por su lado, consigue escapar de los puestos de trabajo del mercado secundario, amén de un entrenamiento y aprendizaje de cara a futuros proyectos empresariales.

Segundo, la solidaridad desde el cliente al empresario, donde se realiza una respuesta al propio grupo, de forma que se aumentan los lazos étnicos y se garantiza una clientela por lealtad. A su vez, el empresario responde abasteciendo de productos "típicamente étnicos" a la comunidad que no encuentran en otros comercios.

Cuando intentamos "medir" la solidaridad étnica desde el empresario al coétnico, necesitamos saber, si cuando se necesitan empleados éstos son de la misma étnia o, en su caso, nacionalidad. Entendiendo que si ocurre esta circunstancia se produce una predilección del empresario hacia su colectivo, con la intención de ayudarle laboralmente. Sin negar el hecho, que contratar a coétnicos es también un reclamo para atraer clientela.

En los casos analizados casi el $100 \%$ de los empleados son coétnicos, teniendo en cuenta que la gran mayoría son familiares. En cualquier caso, la utilización de familiares o coétnicos garantiza una lealtad al empresario, 
al igual que autoexplotación y sacrificio sobre la base de esa solidaridad étnica.

Evidentemente, el apoyo de la red social también se fundamenta de forma explícita, en los procesos de solidaridad étnica invertida, esto es, desde el cliente hacia el empresario. Esta solidaridad ha favorecido el establecimiento y ramificación del empresariado étnico en distintas localidades de la geografía almeriense. Por esto, se puede inferir, a priori, que todas las modalidades de actividad empresarial asociadas a la inmigración están diseñadas hacia una población y clientela eminentemente extranjera. De este modo, el resultado es, hasta cierto punto, predecible: las posibilidades de establecimiento y éxito del conjunto del empresariado étnico pasan por la disponibilidad de un amplio sector de población inmigrada en la zona donde se establezca el negocio.

Así, cuando apreciamos la procedencia de los compradores y clientes habituales de las actividades empresariales étnicas -asociadas a un establecimiento permanente y/o a los mercadillos- observamos cómo los principales compradores divergen, según el tipo de actividad a la que nos estemos refiriendo.

Cuando se trata de establecimientos comerciales con local los compradores son mayoritariamente inmigrados ${ }^{23}(82,7 \%$ del total), de los cuales un $64,8 \%$ son del mismo origen que el empresario o el empleado que "identifica" al comercio.

Por tanto, existe una clara dependencia entre la actividad empresarial étnica y la solidaridad de los clientes. En otras palabras, si no fuera por la afluencia al comercio de población inmigrada -y de manera más concreta, la procedente del mismo origen del empresario y/o empleado-, éste vería mermadas sus ventas considerablemente, hasta incluso desaparecer. Así pues, la dirección de estos comercios se orienta, principalmente, hacia la clientela de origen coétnico que le demanda, preferentemente, productos propios o étnicos.

\footnotetext{
23 Los resultados cambian en la venta ambulante, donde los principales clientes son los autóctonos (véase Arjona, 2004b).
} 


\section{Conclusiones}

La recepción de inmigrantes en la provincia de Almería no se ha producido de manera satisfactoria, han sucedido y existen elementos que nos permiten de hablar de un contexto nada favorable, especialmente en lo laboral y residencial.

El intento de evadirse de las condiciones precarias que ofrece el mercado laboral almeriense ha llevado a algunos inmigrantes a instalar un negocio por cuenta propia. Una vez superadas las trabas jurídicas y sociales, impuestas desde el ámbito nacional hasta el local, los empresarios extracomunitarios han tenido que movilizar gran cantidad de recursos de clase y étnicos, teniendo como base fundamental la utilización de redes. Por ello, cabe destacar, entre todos, dos elementos: capital social y solidaridad étnica.

El capital social está garantizado gracias a un proyecto migratorio de familia extensa y otros miembros de la parentela. Además, en el éxito o fracaso de la empresa, influye, de manera notoria, la utilización de familiares y amigos para trabajar dentro de la misma. Se comienza a configurar, como ya se ha descubierto en otras latitudes, un mercado interno de trabajo.

A través de la solidaridad étnica se conforma un intercambio de ayuda económica que hace posible un mayor afianzamiento de los lazos étnicos, repercutiendo positivamente en el proyecto empresarial. Esta se produce; en primer lugar, desde el empresario al cliente ofreciendo productos típicamente étnicos o empleándolo en su negocio y, en segundo lugar, desde el coétnico al empresario a través de su fidelidad en la compra de sus productos.

\section{Bibliografía}

Arango, J. (1993). “El 'sur' en el sistema migratorio europeo. Evolución reciente y perspectivas", en Política y Sociedad, no 12, págs. 7-20.

Arjona, A. (2004a). "La iniciativa empresarial de los inmigrados. Una aproximación a la economía étnica en Almería", en F. Checa, J. C. Checa y A. Arjona (eds.), Inmigración y derechos humanos. La integración como participación social, Barcelona: Icaria, págs. 149-180. 
- (2004b). Inmigración y mercado de trabajo. El caso de la economía étnica. Tesis Doctoral, Universidad de Almería.

Avellá, L. (1995). “Competencia y complementariedad en el mercado de trabajo entre nacionales e inmigrantes", en Seminario de Inmigración, empleo e integración social, Santander, UIMP.

Bailey, T. y Waldinger, R. (1991). “Primary, secondary and ethnic labor markets: a training system approach", en American Sociological Review, no 56, págs. 432-445.

Beltrán, J. (2000). “Empresa familiar, Trabajo, redes sociales y familia en el colectivo chino", en Ofrim/Suplementos no 6, págs. 29-154.

Berger, B. (1991). The culture of entrepreneurship. San Francisco: ICS Press.

Blaschke, J. y Ersoz, A. (1986). "The Turkish economy in West Berlin”, en International Small Business J ournal, no 4, págs. 38-45.

Body-Gendrot, S. y Ma Mung, E. (1992). “Entrepreneurs entre deus mondes" en Review Européenne des Migrations Internationales, Spécial Issue, no 1 .

Boissevain, J. (1992). "Les entreprises ethniques aux Pais-Bas", en Revue Européenne des Migrations Internationales, vol. 8, № 1, págs. 97-106.

Bonacich, E. (1976). “Advanced capitalism and black/white Race Relations in the United States: a split labor market interpretation", en American Sociological Review, vol. 41, págs. 34-51.

- (1979). "The past, present, and future of split labor market theory", en Research in Race an Ethnic Relations, vol. 1, págs. 17-64.

- (1993). "The other side of ethnic entrepreneurship: a dialogue with Waldinger, Aldrich, Ward and associates", en International Migration Review, no 27, págs. 685-702.

Bonacich, E. y Modell, J. (1980). The Economic Basis of Ethic Solidarity in the Japanese American Community. Berkeley: University of California Press.

- (1981). The economic basis of ethic ethnic solidarity. Los Angeles: University of California. 
Bourdieu, P. (1991). Language and symbolic power. Cambridge: Harvard University.

Cachón, L. (1997). “Segregación sectorial de los inmigrantes en el mercado de trabajo en España”, en Cuadernos de Relaciones Laborales, no 10, págs. 49-73.

Cebrián, J. A. y Bodega, M. I. (2004). “La iniciativa empresarial del inmigrante", en Economistas, no 99, págs. 116-122.

Checa, F. (1995). “Oportunidades socioeconómicas en el proceso migratorio de los inmigrados africanos en Almería", en Agricultura y Sociedad, no 77, págs. 41-82.

Colectivo IOÉ (1995ạ). La discriminación laboral a los trabajadores inmigrantes en España. Estudios sobre migraciones internacionales. Ginebra: OIT, Departamento de Empleo.

- (1995b). “Discriminación contra trabajadores marroquíes en el acceso al trabajo" en La discriminación laboral a los trabajadores inmigrantes en España, Ginebra, OIT.

- (1998). Inmigración y trabajo. Trabajadores inmigrantes en el sector de la construcción. Madrid: Observatorio Permanente de la Inmigración, Instituto de Migraciones y Servicios Sociales (IMSERSO).

Coleman, J. (1988). "Social capital in the creation of human capital", en American J ournal of Sociology, no 94, págs. 103-121.

Engstrom, D. y Mccready, W. (1990). Asian immigrant entrepreneurs in Chicago. Chicago: University of Chicago.

Granovetter, M. (1973). "The strength of weak ties", en American Journal Sociology, vol. 78, no6, págs. 1360-1380.

Herranz, Y. (1996). Formas de incorporación laboral de la inmigración latinoamericana en Madrid. Importancia del contexto de recepción. Tesis Doctoral, Universidad Autónoma de Madrid.

Hoggart, K; Mendoza, C. (1999). “African immigrant workers in Spanish agriculture", Sociologia Ruralis, no 39, págs. 538-562.

Jiobu, R. (1998). Ethnicity and assimilation. Albany: State University of New York. 
Kloosterman, R. (2000). "Immigrant entrepreneurship and the institutional context, A theoretical exploration", en Jan Rath, (ed.), Immigrant businesses, the economic, political and social environment. London: Macmillan, págs 90-106.

Kloosterman, R. y Rath, J. (2001). “Immigrant entrepreneurs in advanced economies, mixed embeddedness further explored", Journal of Ethnic and Migration Studies, no 27, págs. 189-201.

Kloosterman, R., van der Leun, J. y Rath, J. (1998). “Mixed Embeddedness, (In)formal Economic Activities and Immigrant Businesses in the Netherlands", en International Journal of Urban and Regional Research, no 23, págs. 252-266.

Light, I. (1971). Ethnic enterprise in America, Berkeley: University of California Press.

Light, I. y Bonacich, E. (1988). Immigrant Entrepreneurs. Koreans in Los Angeles, 1965-1982. Berkeley: University of California Press.

Light, I. y Gold, S. (2000). Ethnic Economies. San Diego: Academic Press. Light, I. y Karageorgis, S. (1994). "The ethnic economy", en N. Smelser y R. Swedberg (edit.), The handbook of economic sociology, New York, Russell Sage Foundations, págs. 647-671.

Light, I., Sabagh, G., Bozorgmehr, M. y Der-Martirosian, C. (1994).“Beyond the Ethnic enclave economy", en Social Problems, no 1, págs. 65-80.

Logan, J. R., Alba, R. D. y McNULTY, T. L. (1994). “Ethnic Economies in Metropolitan Regions, Miami and Beyond", en Social Forces, no 72, págs. 691-724.

Ma Mung, E. (1994). “L'entreprenariat ethnique en France”, en Sociologie du Travail, no 2, págs. 185-209.

Martínez, F. y otros (1996). Africanos en Andalucía. Necesidades y recursos. Sevilla: Universidad de Sevilla, Junta de Andalucía.

Macdonald, J. S. y L. D. Macdonald (1964). "Chain Migration, Ethnic Neighbourhood Formation and Social Networks." Milbank Memorial Fund Quaterly 42(1): 82-97. 
Maya, I. (1999). Análisis de los recursos de apoyo social de los inmigrantes africanos y latinoamericanos en Andalucía. Tipología de redes y proceso de adaptación. Tesis Doctoral, Departamento de Psicología Social, Universidad de Sevilla.

- (2002). "Tipos de redes personales de los inmigrantes y adaptación psicológica" en Revista hispana para el análisis de redes sociales, vol.1, pp.1-56.

Molina, J.L. ( 1995). “Análisis de redes y cultura organizativa: una propuesta metodológica, en Reis, no 71-71, págs. 249-263.

- (2000). El análisis de redes sociales. Una introducción. Bellaterra: Barcelona

Mottura, G. (1992). "Forme della presenza extracomunitraia nella agricoltora italiana: resultadi di una prima exploracione", Ponencia defendida en el curso Trabajadores inmigrantes en la agricultura mediterránea, Valencia.

Munnell, A.; Lynn, E.; Mceneiney, J.; Tootell, G. (1996). “Mortage lending in Boston: interpreting HMDA data", en American Economic Review, no 86, págs. 25-53.

Osterman, P. (1976). The labor market for young men. MIT, Department of economics and Urban Studies.

- (1980). Getting started: the youth labor market. Cambridge, MT Press.

Pierce, J. (1974). Negro business and businessmen education. New York, Harper and Brothers.

Piore, M. (1974). "Notes for a theory of labor market stratification", en R. C. Edwards M. Relch y D. M. Gordon (edits.), Labor market Segmentation, Lexington Mass, Lexington Books, págs. 125-149.

Portes, A. (1998). "Social Capital, Its Origins and Applications in Modern Society", en Annual Review of Sociology, no 24, págs. 1-24.

Portes, A. y Bach, R. (1985). Latin Journey. Cuban and Mexican Immigrants in the United States. Berkeley: University of California Press. 
Portes, A. Böröcz, J. (1992). “Inmigración contemporánea: perspectivas teóricas sobre sus determinantes y modos de acceso", en Alfoz, no 91-92, págs. 20-33.

Portes, A. y Manning, R. D. (1986). "The immigrant enclave: Theory and empirical examples", en J. Nagel y S. Olzak (edits.), Comparative Ethnic Relations, New York, Academic Press, págs.47-68.

Pugliese, E. (1992). "L'immigraziones in Italia: un confronto tra nord e sud", Ponencia defendida en el curso Trabajadores inmigrantes en la agricultura mediterránea, Valencia.

Requena Santos, F. (1990/91) "Redes sociales y mecanismos de acceso al mercado de trabajo", en Sociología del Trabajo, no 11, págs. 117-140.

-(1991) Redes sociales y mercado de trabajo. Elementos para una teoría del capital relacional. Madrid: CIS.

Simon, G. (1990). "Immigrant entrepreneurs in France: A European overview", en Institute for Social Science Research, Vol. 5, Paper 9.

Speck, R. y Attneave, C. (1974). Redes familiares. Buenos Aires: Amorrotu.

Stalker, P. (2000). Workers without frontiers. The impact of globalization on international migration. Colorado: Lynne Rienner Publishers.

Tienda, M. y Raijman, R. (2000). “Immigrants' Income Packaging and Invisible Labor Force Activity", en Social Science Quarterly, no 81, págs. 291-310.

Trinidad, M. L. (2003). “El trabajo por cuenta propia de los extranjeros en España", en Migraciones, № 13, págs. 61-106.

Waldinger, R. (1985). "The making of an immigrant niche", en International Migration Review, no 1, págs. 1-30.

- (1993). "The ethnic enclave debate revised", en International Journal of Urban and Regional Research, no 13, págs. 34-51.

- (1994). "The making of an immigrant niche", en International Migration Review, no 28, págs. 3-30.

Waldinger, R., Aldrich, H. y Ward, R. (eds.) (1990). Ethnic entrepreneurs. London: Sage Publications. 